\title{
Surface Enhanced Raman Scattering Spectroscopy (SERS) Analysis of Ink Pens in Forensic Usage
}

\author{
Keseven Lakshmanan ${ }^{1}$, Balamurugan Thirumalraj*2 and Chelladurai Karuppiah*3 \\ ${ }^{1}$ Graduate Institute of Applied Science Technology, Taiwan \\ ${ }^{2}$ Department of Chemical Engineering, Taiwan \\ ${ }^{3}$ Battery Research Center of Green Energy, Ming Chi University of Technology, Taiwan
}

*Corresponding author: Balamurugan Thirumalraj and Chelladurai Karuppiah, Department of Chemical Engineering, Battery Research Center of Green Energy, Ming Chi University of Technology, Taiwan

\begin{abstract}
ARTICLE INFO
Received: 蔧 March 18, 2019

Published: March 25, 2019

Citation: Keseven Lakshmanan, Balamurugan Thirumalraj, Chelladurai Karuppiah. Surface Enhanced Raman Scattering Spectroscopy (SERS) Analysis of Ink Pens in Forensic Usage Biomed J Sci \& Tech Res 16(2)-2019. BJSTR. MS.ID.002837.
\end{abstract}

Keywords: Nano $\mathrm{Cu}$ on Pencil Graphite; SERS; Ink Pens; Forensic Analysis

\section{ABSTRACT}

The ink pens are very important in forensic questioned documents analysis in developing countries while the chances of fraud, manipulate documents and entry of the date of birth and death. In this mini review, fountain blue, red, green pen inks were used in the above-mentioned documents which have been discriminated by Raman spectroscopy (henceforth referred to as RS) and Surface-enhanced Raman scattering spectroscopy (henceforth referred to as SERS). RS is becoming major importance tool in forensic analysis because it is non-invasive, non-destructive analytical method allowed to sample examined without sample preparation. Raman spectra for different inks on questioned documents are measured at the uniform condition for all the samples. The spectral results are subjected to background correction and smoothening. The pre-processed spectral data are used for carrying out principle compound analysis (PCA). Through, the combination of RS and a statistical PCA method, we could able to differentiate among very similar ink types in the documents. Besides, the RS and PCA helped in identifying correctly the unknown ink pen.

Moreover, the SERS was used to enhance the signals for less sensitive ink samples and quench the fluorescence effect. In this review we discuss about the electrodeposited copper on the pencil graphite surface as the SERS substrate for the forensic analysis of ink pens. This above-proposed method seems ease to make SERS substrate and low price which can be magnificently enhanced the plasmonic resonance effect. Hence, the substrate can also be used directly for a wide range of chemical and biological samples analysis not only for pen inks.

\section{Introduction}

This mini review describes to distinguish the same colors inks in the questioned documents and in other documentary departments. In order to, copying and mala fide alteration of legal and other essential documents are becoming increasingly sophisticated with the up gradation of technology. Identifying such malpractices is of utmost importance to various stakeholders. The pen inks have been extensively used as an instrument of writing for more than 10 decades. In our public existence, it is regularly used to sign official documents. In forensic questioned document examination, criminal cases concerning inaccurate or rewritten documents come across for examination and, therefore, the way for detection and dating of pen ink has a vital role in that field [1]. Among various forms of forging document, altering with a different ink or dye is as usual in routine life. In most cases, the capability to discriminate between different inks can be very helpful. Backdate amendment in a document by writing with pen inks containing indistinguishable (the same) color may show the dissimilar composition of dye. Thus, the comparison of inks may establish a connection between two spec- 
imens in the case of forgery [2]. However, it is not easy to analyze inks by nondestructive techniques. So, the distinction between inks is important for forensic document analysis where the document under investigation was suspected of forgery by ink.

In order to evaluate the forgery, Raman spectroscopy (RS) is an optical technique that can be used in a non-destructive manner for the forensic investigation of documents. So that, the questioned documents inks contain fluorescence compound, less amount of forensic samples and less sensitivity dye. Thus, the RS studies were showed the spectral saturation and most of the time it doesn't show anything from the above-mentioned forensic samples. However, the fluorescence interference and low sensitivity is a common problem, resulting poor analytical performance. Hence, Surface-enhanced Raman spectroscopy (SERS) is becoming an effective tool to enhance the spectrum signals. It can quench the fluorescence effect and provide highly sensitive signals, all of which ultimately lead to improved detection limits. Thus, the SERS is a highly sensitive and selective variant of the Raman spectroscopy [3,4]. In SERS, we need to use a suitable substrate for sample adsorption. Generally, the substrate has been made up of metals, especially, noble metals for effectively adsorbed on metallic surface, usually gold, silver, and copper has been used as substrate for sample preparation. Mainly, the surface becomes SERS active and fluorescence quenched, and the suitable to produce the Raman signal which is enhanced by the electromagnetic interaction of the light with the metals. Additionally, the improvement can be up to $10^{\wedge} 6$ in scattering efficiency over the normal Raman scattering [5].

This interaction can be produced large amplification of the laser field through excitations generally known as "Plasmon resonances" [6]. There have been many reports on SERS using noble metal nanoparticles and nanostructures as substrates in the literature. Notably, Ramanathan et al reported the pencil graphite over the metallic nanoparticles by electrodeposition is a novel and new substrate for SERS $[7,8]$. The electrodeposited nanostructures on pencil graphite has good mechanical, thermal and electrical conducting properties while compared with another substrate. Noble metals have been the most popular for the choice of electrodeposition process owing to their remarkable outstanding properties. Copper is relatively low cost, retention of high stability and good catalytic activity and serves as a plausible alternative for platinum, gold, and silver. Although, the $\mathrm{Cu}$ nanostructures have tendency to undergo atmospheric oxidation easily $[9,10]$. Surprisingly, the electrodeposited metals, particularly $\mathrm{Cu}$, on pencil graphite have not been used as SERS substrate. Here, we introduce the use of electrodeposited $\mathrm{Cu}$ nanostructures on pencil graphite as a promising substrate for SERS. However, Cu can be used as a SERS substrate even though the formation of oxides on the $\mathrm{Cu}$ surface [10]. Different brands of inks were collected in the questioned documents examined by Raman spectroscopy and SERS which were measured at the uniform condition for all the samples.

The spectral results were subjected to background correction and smoothening (FFT filter). The pre-processed spectral data were used for carrying out PCA analysis. Through the combination of RS, Pearson's correlation calculation and PCA method, we were able to differentiate among very similar ink types in documents. PCA transforms dataset with goal representing data of changes in few uncorrelated axes called principal components (PCs). PCA provides a dimension reduction that has the advantage of facilitating the identification and visualization of the data structure. The pathway for SERS detection using Nano cu on pencil graphite was shown in Scheme 1. The PCs are a linear combination of the original variables and describe the data variance in decreasing order. The PC1 indicates the direction of higher variability of the data and the PC2 indicates the second highest variability in the data uncorrelated to the first one and so on. The Pearson's correlation matrix calculations of all the possible pairwise combination were collected from XL state 2018.1 version while the PCA was performed using UNSCRAMBLER software. These reports indicates that the combination of RS and suitable substrate of SERS with statistical method (PCA) seems to distinguish the different ink pens of the same color of forensic analysis of questioned documents

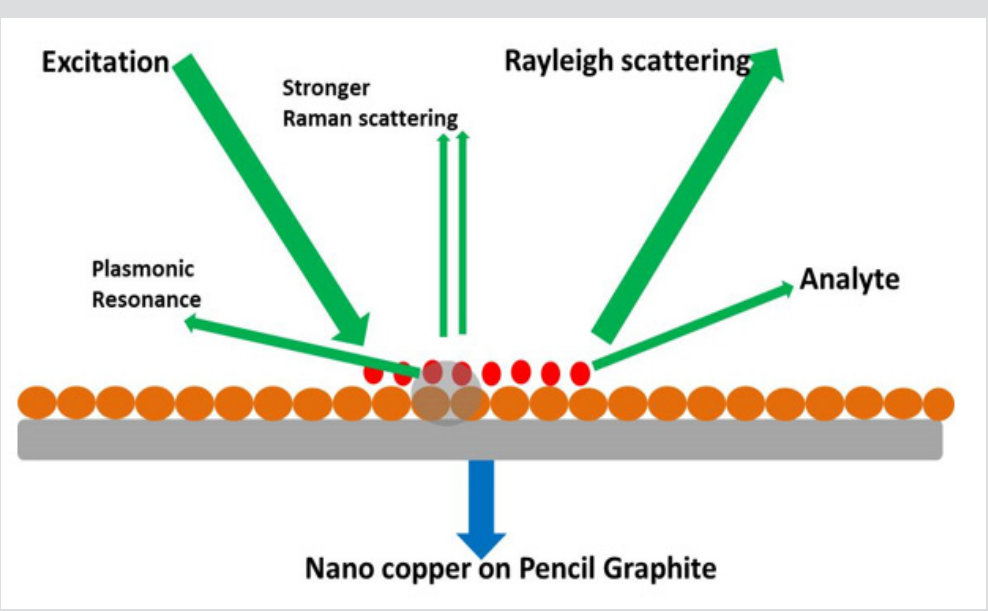

Scheme 1: Schematic illustration of SERS pathway using Nano copper on pencil graphite. 


\section{Conclusion}

In this report, we successfully introduced a unified concept of RS and SERS with PCA to differentiate the ink pens in the questioned documents of forensic analysis. Based on the results, we conclude that the great advantage of RS relied on its non-invasive nature. However, more efficient identification was achieved by SERS, through the electrodeposition of $\mathrm{Cu}$ nanoparticles on pencil graphite, which can act as a potential substrate for SERS studies. This study also revealed the combination of RS and PCA has promising fast non-destructive tool to differentiate very similar inks in documents. In addition, the RS and PCA were successfully applied for the source determination.

\section{Acknowledgement}

This work was supported by the Ministry Science and Technology and the Ministry of Education of Taiwan (Republic of China).

\section{References}

1. Wang XF, Yu J, Xie MX, Yao YT, Han J, et al. (2008) Identification and dating of the fountain pen ink entries on documents by ion-pairing highperformance liquid chromatography. Forensic Science International 180(1): 43-49.

2. Egan JM, Hagan KA, Brewer JD (2005) Forensic analysis of black ballpoint pen inks using capillary electrophoresis. Forensic Science Communications $7(3)$.

\section{ISSN: 2574-1241}

DOI: 10.26717/BJSTR.2019.16.002837

Thirumalraj B, Karuppiah C. Biomed J Sci \& Tech Res

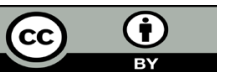

This work is licensed under Creative Commons Attribution 4.0 License

Submission Link: https://biomedres.us/submit-manuscript.php
3. Rodger C, Dent G, Watkinson J, Smith WE (2000) Surface-Enhanced Resonance Raman Scattering and Near-Infrared Fourier Transform Raman Scattering as in Situ Probes of Ink Jet Dyes Printed on Paper. J Appl Spect 54(11): 1567-1576.

4. Ru E, Etchegoin P (2009) Principles of surface-enhanced raman spectroscopy. J Am Chem Soc 131: 14466-14472.

5. Guillot N, Lamyde M, Chaplle LA (2012) The electromagnetic effect in surface enhanced raman scattering: Enhancement optimization using precisely controlled nanostructures. J Quant Spect And Rel Trans 113(18): 2321-2333.

6. Gao Y, Yang N, Yu T, Jiang L, Yin P, et al. (2017) Ultra-thin Au tip structure: a novel SERS substrate for in situ observation of a p-aminothiophenol surface-catalysis reaction. RSC Adv 7: 4541-4546.

7. Arathi PJ, See mesh B, Reddy GRK, Kumar PS, Ramanathan V, et al. (2016) Disulphide linkage: To get cleaved or not? Bulk and Nano copper-based SERS of cysteine. Mater Sci Eng C 196: 229-232.

8. Majidi MR, Zeynali KA, Hafezi B (2016) Electrocatalytic oxidation and determination ofceftriaxone sodium antibiotic in pharmaceutical samples on a copper hexacyanoferrate nanostructure. Anal Methods 3(3): 646-652.

9. Reddy GRK, Kumar PS (2017) Template electrodeposition of highperformance copper oxide nanosensors for electrochemical analysis of hydrogen peroxide. Mater Sci Eng C 75: 1480-1488.

10. Gawande MB, Goswami A, Felpin FX, Asefa T, Huang X, et al. (2016) Cu and $\mathrm{Cu}$-based nanoparticles: Synthesis and applications in catalysis. Chem Rev 116(6): 3722-3811.

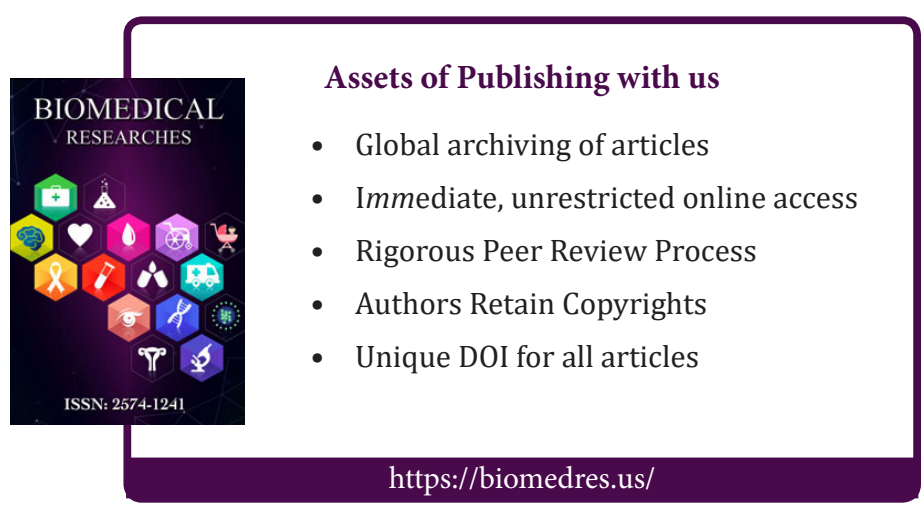

